\title{
Forced Arterial Suction Thrombectomy with the Penumbra Reperfusion Catheter in Acute Basilar Artery Occlusion: A Retrospective Comparison Study in 2 Korean University Hospitals
}

\author{
Y.-I. Eom, 이.-H. Hwang, J.M. Hong, J.W. Choi, Y.C. Lim, D.-H. Kang, Y.-W. Kim, Y.-S. Kim, S.Y. Kim, and J.S. Lee
}

\begin{abstract}
BACKGROUND AND PURPOSE: A performance of forced arterial suction thrombectomy was not reported for the treatment of acute basilar artery occlusion. This study compared revascularization performance between intra-arterial fibrinolytic treatment and forced arterial suction thrombectomy with a Penumbra reperfusion catheter in patients with acute basilar artery occlusion.
\end{abstract}

MATERIALS AND METHODS: Fifty-seven patients with acute basilar artery occlusion were treated with intra-arterial fibrinolysis $(n=25)$ or forced arterial suction thrombectomy $(n=32)$. Baseline characteristics, successful revascularization rate, and clinical outcomes were compared between the groups.

RESULTS: Baseline characteristics, the frequency of patients receiving intravenous recombinant tissue plasminogen activator, and mean time interval between symptom onset and femoral puncture did not differ between groups. The forced arterial suction thrombectomy group had a shorter procedure duration (75.5 minutes versus 113.3 minutes, $P=.016$ ) and higher successful revascularization rate ( $88 \%$ versus $60 \%, P=.017)$ than the fibrinolysis group. Fair outcome, indicated by a modified Rankin Scale $0-3$, at 3 months was achieved in $34 \%$ of patients undergoing forced arterial suction thrombectomy and $8 \%$ of patients undergoing fibrinolysis $(P=.019)$, and the mortality rate was significantly higher in the fibrinolysis group ( $25 \%$ versus $68 \%, P=.001)$. Multiple logistic regression analysis identified the forced arterial suction thrombectomy method as an independent predictor of fair outcome with adjustment for age, sex, initial NIHSS score, and the use of intravenous recombinant tissue plasminogen activator (odds ratio, 7.768; 95\% Cl, 1.246-48.416; $P=.028$ ).

CONCLUSIONS: In acute basilar artery occlusion, forced arterial suction thrombectomy demonstrated a higher revascularization rate and improved clinical outcome compared with traditional intra-arterial fibrinolysis. Further clinical trials with the newer Penumbra catheter are warranted.

ABBREVIATIONS: BAO = basilar artery occlusion; IA = intra-arterial; FAST = forced arterial suction thrombectomy

$\mathbf{T}$ he prognosis for acute basilar artery occlusion (BAO) is dismal. ${ }^{1,2}$ Early recanalization is one of the most important prognostic factors for an improved outcome in BAO., Intravenous infusion of recombinant tissue plasminogen activator is, however, not fully effective for the recanalization of acute BAO. ${ }^{5,6}$

Endovascular treatment is an emerging therapeutic option for acute BAO. Previously, local infusion of fibrinolytic agents was

Received March 28, 2014; accepted after revision May 28.

From the Departments of Neurology (Y.-I.E., J.M.H., J.S.L.) and Radiology (J.W.C., S.Y.K.), Ajou University Medical Center, Suwon, South Korea; Departments of Neurology (Y.-H.H., Y.-W.K.), Neurosurgery (D.-H.K.), and Radiology (D.-H.K., Y.-W.K., Y.-S.K.), Kyungpook National University Hospital, Daegu, South Korea; and Department of Neurosurgery (Y.C.L.), Ajou University Hospital, Daegu, South Korea.

This work was supported by the new faculty research fund of the Ajou University School of Medicine (J.S. Lee).

Please address correspondence to Jin Soo Lee, MD, PhD, Department of Neurology, Ajou University Medical Center, Suwon, South Korea; e-mail: jinsoo22@gmail.com

http://dx.doi.org/10.3174/ajnr.A4045 the only available therapy. ${ }^{2,7,8}$ Although intra-arterial fibrinolysis achieved a much higher recanalization rate than intravenous rtPA, clinical outcome did not differ between the treatments. ${ }^{9}$ Recently, several mechanical thrombectomy devices have been developed and applied in clinical studies. ${ }^{10-13}$ However, thus far, no device has been proved to improve clinical outcomes, and the optimal strategy for acute BAO treatment has not yet been established.

The Penumbra System (Penumbra, Alameda, California) is an endovascular device designed to reduce clot burden in acute ischemic stroke due to large cerebral artery occlusion. Although many clinical trials have been conducted using the device, data concerning the use of the Penumbra System in acute BAO are still scarce. ${ }^{10}$ Forced arterial suction thrombectomy (FAST), which is a modification of the standard Penumbra System, is used as a mechanical recanalization method for ischemic stroke. ${ }^{14,15}$ Unlike the standard Penumbra System, the FAST method involves only a reperfusion catheter. Negative 


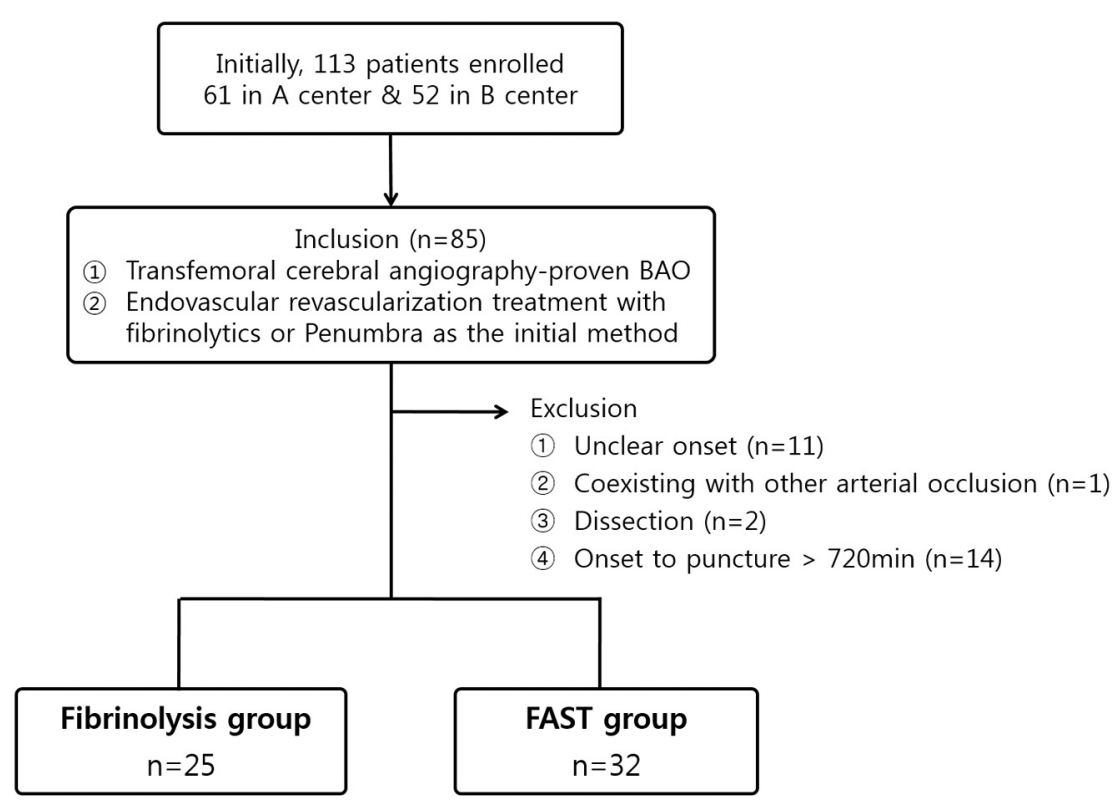

FIG 1. Flow diagram of the current study.

Table 1: Baseline demographics, neurologic deficits, and hyperacute treatment status

\begin{tabular}{lccc}
\hline & Fibrinolysis Group & FAST Group & $P$ Value \\
\hline No. of patients & 25 & 32 & \\
Age (yr) (mean) & $66.6 \pm 10.2$ & $68.3 \pm 10.7$ & .549 \\
Male & $21(84 \%)$ & $22(69 \%)$ & .184 \\
Hypertension & $11(44 \%)$ & $21(66 \%)$ & .103 \\
Diabetes mellitus & $7(28 \%)$ & $9(28 \%)$ & .992 \\
Atrial fibrillation & $8(32 \%)$ & $11(34 \%)$ & .850 \\
NIHSS score on admission (mean) & $19.9 \pm 8.1$ & $19.8 \pm 8.7$ & .965 \\
TOAST classification & & & .737 \\
Atherosclerosis & $14(56 \%)$ & $15(47 \%)$ & \\
Cardioembolism & $7(28 \%)$ & $12(37 \%)$ & \\
Mixed or unknown & $4(16 \%)$ & $5(16 \%)$ & \\
Use of intravenous rtPA & $7(28 \%)$ & $12(36 \%)$ & .35 \\
Time from onset to puncture (min) (mean) & $297.0 \pm 125.1$ & $281.4 \pm 138.3$ & .662 \\
Primary endovascular treatment & & & \\
Urokinase & $22(88 \%)$ & - & \\
rtPA & $2(8 \%)$ & & \\
Tirofiban & $1(4 \%)$ & & \\
Penumbra & - & $32(100 \%)$ & \\
Rescue treatment methods & & & \\
Stenting & $7(28 \%)$ & $6(18.8 \%)$ & \\
Tirofiban & $2(8 \%)$ & $2(6.3 \%)$ & \\
Abciximab & $2(8 \%)$ & - & \\
Penumbra & $2(8 \%)$ & & \\
Solitaire & - & $3(9.4 \%)$ & \\
\hline
\end{tabular}

Note:-TOAST indicates Trial of Org 10172 in Acute Stroke Treatment. ${ }^{34}$

pressure via forceful pulling of a syringe can keep an embolus aspirated at the catheter tip. This method has been reported to be simpler and faster than the standard Penumbra System and has been applied clinically. ${ }^{14,16,17}$

We postulated that the FAST method may improve the clinical outcome of acute BAO compared with the traditional endovascular treatment method of local fibrinolysis. In the present study, we evaluated the revascularization performance and clinical outcomes between intra-arterial (IA) fibrinolytic treatment and the FAST method and compared them with the Penumbra reperfusion catheter in patients with acute BAO.

\section{MATERIALS AND METHODS Study Population}

This retrospective study involved registry data bases and additional imaging analyses. All patients or their caregivers gave informed consent for each endovascular treatment. From registries of 2 university hospitals in Korea, 113 consecutive patients who had BAO with or without continuous vertebral artery occlusion and underwent revascularization treatments between March 2006 and June 2013 were enrolled (Fig 1). Among them, those who had transfemoral cerebral angiography-proved BAO and underwent IA revascularization with either fibrinolysis or mechanical thrombectomy by using FAST as the initial IA treatment method for acute BAO were included. Patients were excluded under the following conditions: 1) their onset of symptoms was unclear, 2) another cerebral artery was occluded, 3) onset-to-puncture time exceeded 720 minutes, or 4) their stroke etiology was dissection. We grouped patients into a fibrinolysis group and a FAST group, depending on the initial method of endovascular treatment. Other endovascular methods for rescue therapy were permitted.

\section{Protocol}

Brain parenchyma and cervicocerebral arteries were evaluated by CT or MR imaging as early as possible before IA treatment. The BAO as a treatment target was confirmed by selective cerebral intra-arterial digital subtraction angiography. Patients received either endovascular revascularization treatment following intravenous thrombolysis or endovascular treatment alone. Local intra-arterial fibrinolysis was performed by using an infusion microcatheter inserted into the middle of the clots. Fibrinolytic agents included rtPA, urokinase, abciximab, and tirofiban. All FAST methods were performed by using the Penumbra aspiration catheter (first-generation). Using a transfemoral approach, we placed the guide catheter into the dominant or most navigable vertebral artery. After we placed the reperfusion catheter immediately proximal to the clot, we applied negative pressure at the distal tip by continuous suctioning with a $50-\mathrm{mL}$ syringe. The catheter was then smoothly pulled back. The interventional procedure was terminated when recanalization was achieved or according to a consensus between neurologists and neurointerventionists. 


\begin{tabular}{|c|c|c|c|}
\hline & Fibrinolysis Group & FAST Group & $P$ Value \\
\hline Procedure time (min) (mean) & $113.3 \pm 65.4$ & $75.5 \pm 42.2$ & .016 \\
\hline Time from onset to final angiography (min) (mean) & $419.5 \pm 144.7$ & $364.7 \pm 145.2$ & .163 \\
\hline Successful revascularization & $15(60 \%)$ & $28(88 \%)$ & .017 \\
\hline Intracranial hemorrhage & & & .157 \\
\hline Hemorrhagic transformation type 1 & $7(29 \%)$ & $4(13 \%)$ & \\
\hline Hemorrhagic transformation type 2 & - & - & \\
\hline Parenchymal hematoma type 1 & - & - & \\
\hline Parenchymal hematoma type 2 & $1(4 \%)$ & - & \\
\hline $\mathrm{SAH}$ & $2(8 \%)$ & $2(6 \%)$ & .797 \\
\hline NIHSS score at discharge (mean) & $20.2 \pm 11.8$ & $9.2 \pm 12.8$ & .066 \\
\hline Fair outcome at 3 months & $2(8 \%)$ & $11(34 \%)$ & .019 \\
\hline Mortality & $17(68 \%)$ & $8(25 \%)$ & .001 \\
\hline Successful revascularization with the primary stand-alone technique & 13 of $21(61.9 \%)$ & 24 of $26(92.3 \%)$ & .011 \\
\hline Procedure time of the primary stand-alone technique (min) (mean) & $91.1 \pm 44.6$ & $66.2 \pm 34.0$ & .037 \\
\hline Fair outcome at 3 months with the primary stand-alone technique & 1 of $21(4.8 \%)$ & 10 of $26(38.5 \%)$ & .007 \\
\hline
\end{tabular}

\section{Outcome Measurements}

Baseline patient data, including vascular risk factors, stroke etiology, laboratory and imaging variables, and initial neurologic severity scales, were retrieved from each stroke registry data base and electronic health records. Imaging data were collected from the PACS of each hospital. Procedural duration was defined as the time between the placement of a guide catheter proximal to the target artery and final angiography of the target artery. Revascularization was measured by the Thrombolysis in Cerebral Infarction score. Successful revascularization was defined as a TICI score of $2 \mathrm{~b}$ (perfusion $\geq 50 \%$ ) or 3 . Functional outcomes were measured by using the modified Rankin Scale and mortality at 3 months. Fair outcome at 3 months was defined as an mRS score of $0-3$.

\section{Statistical Analysis}

Univariate analyses were performed by using the $t$ test for continuous variables and the $\chi^{2}$ test for nonparametric variables. Logistic regression analysis was used to evaluate whether the FAST method was an independent predictor of fair outcomes at 3 months. Two-sided $P$ values $<.05$ were considered significant. Statistical analyses were performed by using a commercially available software package (SPSS, Version 17.0 for Windows; IBM, Armonk, New York).

\section{RESULTS}

Ultimately, 57 patients were included in the study: 25 in the fibrinolysis group and 32 in the FAST group. Baseline characteristics were similar between groups (Table 1). Mean age, the frequency of males, and the mean initial National Institutes of Health Stroke Scale score did not differ significantly between groups. The frequency of patients who underwent intravenous rtPA and the mean time interval between symptom onset and femoral puncture also did not differ between groups.

Procedural and clinical outcomes are presented in Table 2. Urokinase was most frequently used for primary IA fibrinolysis (88\%; mean, 240,000 U). Tirofiban and rtPA were used in a small number of patients who underwent primary IA fibrinolysis. In patients who underwent the primary FAST procedure, the Penumbra reperfusion catheter 041 was most frequently used (29 cases), followed by the Penumbra reperfusion catheter 032 (3
Modified Rankin Scale

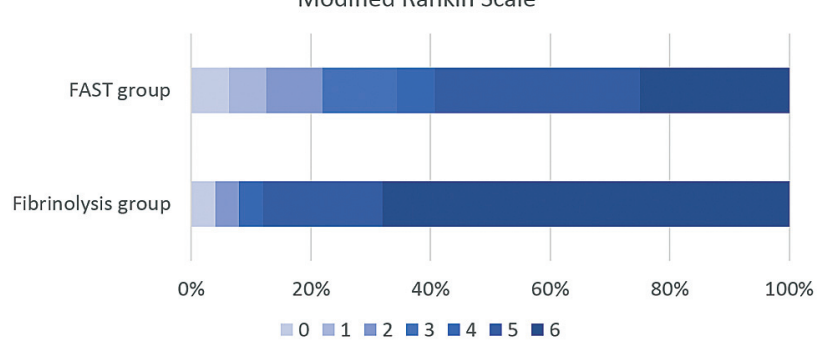

FIG 2. Modified Rankin Scale of each group. Mortality was significantly reduced when patients underwent endovascular treatment with the FAST method compared with local fibrinolysis.

Table 3: Multiple logistic regression model for fair outcome at 3 months

\begin{tabular}{llc}
\hline \multicolumn{1}{c}{ Variables } & \multicolumn{1}{c}{ OR $(95 \% \mathrm{CI})$} & $\boldsymbol{P}$ Value \\
\hline Age & $0.951(0.881-1.027)$ & .199 \\
Sex & $0.135(0.013-1.389)$ & .092 \\
Initial NIHSS score & $0.914(0.828-1.009)$ & .073 \\
Intravenous rtPA & $3.749(0.685-20.512)$ & .128 \\
FAST method & $7.768(1.246-48.416)$ & .028 \\
Successful revascularization & $4.339(0.266-70.698)$ & .303 \\
\hline
\end{tabular}

cases). Furthermore, the Penumbra reperfusion catheter 026 (2 cases) was used only for rescue therapy in the fibrinolysis group. A separator was not used in any of the cases in both the hospitals. The median number of passes performed with the Penumbra reperfusion catheter was 2 (interquartile range, 1-3). The mean procedural duration was significantly shorter in the FAST group than in the fibrinolysis group (76 minutes versus 113 minutes, $P=$ .016). Successful revascularization was achieved at a higher rate in the FAST group than in the fibrinolysis group ( $88 \%$ versus $60 \%$, $P=.017)$. The rate of fair outcome at 3 months was significantly higher in the FAST group than in the fibrinolysis group (36\% versus $8 \%, P=.015$ ), while the mortality rate was significantly higher in the fibrinolysis group (23\% versus $68 \%, P=.001$; Fig 2 ). A multiple logistic regression model revealed that the FAST method was an independent predictor of fair outcome with adjustment for age, sex, initial NIHSS score, and the infusion of intravenous rtPA (odds ratio, 7.768; 95\% CI, 1.246-48.416; $P=$ .028; Table 3).

Notably, our cohort demonstrated unique characteristics concerning etiology, with atherosclerosis accounting for $50 \%$ of pa- 
Table 4: List of revascularization treatment studies for acute basilar artery occlusion

\begin{tabular}{|c|c|c|c|c|c|c|c|c|c|c|c|}
\hline Reference & Method & $\begin{array}{c}\text { No. of } \\
\text { Patients }\end{array}$ & Age (yr) & $\begin{array}{l}\text { NIHSS on } \\
\text { Admission }\end{array}$ & $\begin{array}{c}\text { Good } \\
\text { Outcome }\end{array}$ & $\begin{array}{c}\text { Fair } \\
\text { Outcome }\end{array}$ & Mortality & $\underset{2-3}{\text { TIMI }}$ & $\begin{array}{l}\mathrm{TICI} \\
2 \mathrm{~b}-3\end{array}$ & sICH & SAH \\
\hline Arnold et al $(2004)^{31}$ & Urokinase & 40 & 58 (median) & 18 (median) & $35 \%$ & $47 \%$ & $42 \%$ & $60 \%$ & - & $5 \%$ & - \\
\hline Lutsep et al $(2008)^{13}$ & Merci & 27 & 58 (median) & 22 (median) & $33 \%$ & $41 \%$ & $44 \%$ & $78 \%$ & - & $19 \%$ & $11 \%$ \\
\hline Roth et al $(2011)^{10}$ & Standard Penumbra System & 12 & 70 (median) & 28 (median) & $33 \%^{\mathrm{a}}$ & $50 \%^{a}$ & $33 \%^{\mathrm{a}}$ & $83 \% \mathrm{~b}$ & - & - & - \\
\hline Mourand et al $(2014)^{30}$ & Solitaire & 31 & 61 (mean) & 38 (median) & $35 \%$ & - & $32 \%$ & - & $74 \%$ & $16 \%$ & - \\
\hline Espinosa de Ruedz et al $(2013)^{28}$ & Solitaire, Trevo & 18 & 68 (mean) & 20 (mean) $^{c}$ & $50 \%$ & - & $22 \%$ & - & $94.4 \%$ & - & $6 \%$ \\
\hline Current study, fibrinolysis & Urokinase, rtPA, tirofiban & 25 & 67 (mean) & 20 (mean) & $8 \%$ & $8 \%$ & $68 \%$ & - & $36 \%$ & $4 \%$ & $8 \%$ \\
\hline Current study, FAST & FAST method & 33 & 68 (mean) & 20 (mean) & $22 \%$ & $35 \%$ & $21 \%$ & - & $79 \%$ & $0 \%$ & $6 \%$ \\
\hline
\end{tabular}

Note:-TIMI indicates Thrombolysis in Myocardial Infarction; sICH, symptomatic intracerebral hemorrhage.

a Scales were evaluated before discharge.

${ }^{b}$ Definition of recanalization was not clearly documented.

' NIHSS score on admission was evaluated only in nonintubated patients (12 of 18).

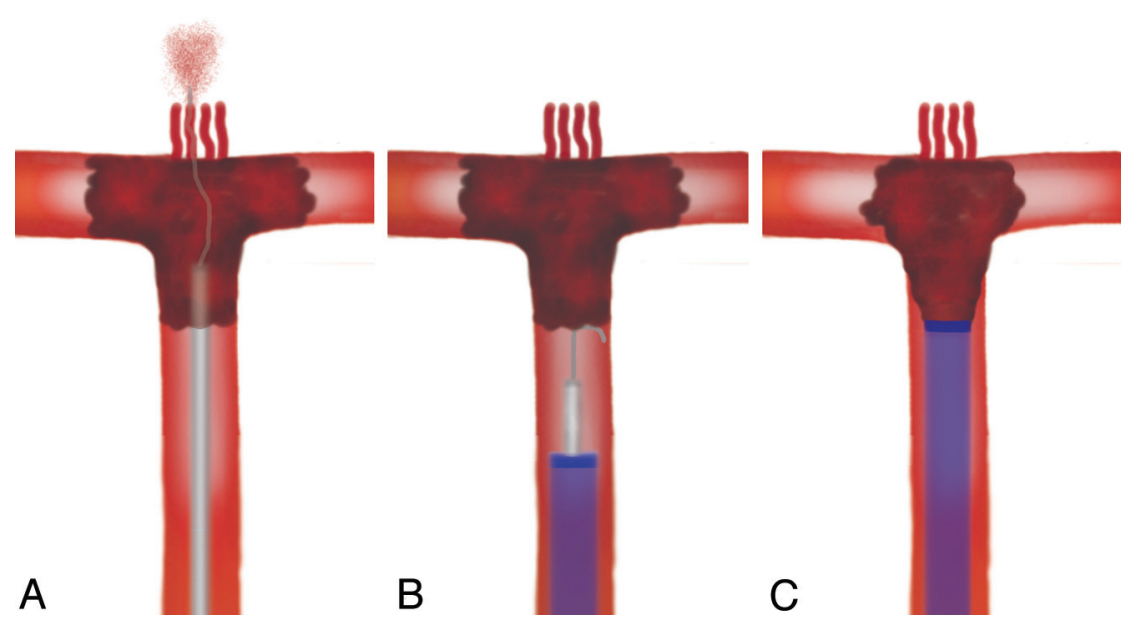

FIG 3. Schematic drawings of endovascular treatment for acute BAO. A, Arterial branches are obscured above the occlusive site, and inadvertent hemorrhagic complications can occur when a microwire and microcatheter must be navigated distal to the occlusive site. $B$ and $C$, The FAST method can avoid this risk because the tip of the Penumbra reperfusion catheter is located only at the proximal end of the clot.

tients. Specifically, intracranial stent placement was performed in $19 \%$ and $28 \%$ of patients in the FAST and fibrinolysis groups, respectively.

\section{DISCUSSION}

In this series, we have demonstrated that the FAST method with the Penumbra aspiration catheter can rapidly and effectively restore blood flow in acute BAO and can improve patient outcome in comparison with the traditional IA fibrinolysis method. Better clinical outcome was attributed to higher performance in terms of the revascularization rate and shorter procedural duration. This study is the first to report the efficacy of FAST with the Penumbra reperfusion catheter for the treatment of acute BAO in comparison with IA fibrinolysis.

Acute BAO is a devastating disease, which has been associated with a poor clinical outcome and a high mortality rate. ${ }^{1,2}$ Treatment with intravenous thrombolysis has been shown to be ineffective for the recanalization of BAO or carotid T occlusion. ${ }^{18,19}$ On the basis of these limitations, IA injection of fibrinolytic agents has been adopted in standard protocols to treat BAO by many stroke centers, and several randomized trials and numerous case series have demonstrated the benefits of this therapy. 2,7,20-22 However, despite the high recanalization rate of intra-arterial fibrinolysis therapy, clinical outcome has not improved. ${ }^{23-25}$ In the past several years, the introduction of mechanical thrombectomy devices has offered a new option for the treatment of large-vessel occlusion, achieving higher recanalization rates and favorable clinical outcomes. $^{5,10,12,13,26,27}$

At the start of the endovascular treatment era for BAO, Hacke et $\mathrm{al}^{2}$ published findings in 1988 demonstrating better outcomes and higher survival rates in patients who underwent IA fibrinolysis compared with those who received conventional treatment. On the basis of that report, IA fibrinolysis has been considered an additional treatment option for patients with acute BAO. Recently, several studies have described the performance and efficacy of new mechanical thrombectomy devices for the treatment of BAO. The Merci retrieval system (Concentric Medical, Mountain View, California) was reported as a feasible option for the revascularization of acute $\mathrm{BAO}$, with a recanalization rate of $68 \% .{ }^{13}$ Several studies using the Solitaire retrieval stent (Covidien, Irvine, California) in acute BAO have also been reported. ${ }^{11,12,26-29}$ The latest studies have achieved revascularization rates of $74 \%-94 \%$ and mortality rates of $22 \%-32 \%$ by using stent-retrieval systems. ${ }^{27,28}$ Previous studies of endovascular treatment for BAO are summarized in Table $4 .^{10,13,28,30,31}$

Limited literature is available on use of the Penumbra system for acute BAO. In one study, 12 patients with acute BAO were treated with the standard Penumbra System, with a recanalization rate of $75 \%$ and a mortality rate of $33 \% .{ }^{10}$ In the present study, a successful revascularization rate of $79 \%$ and a $21 \%$ mortality rate were observed with the FAST method. Compared with traditional IA fibrinolysis, which had a successful revascularization rate of $36 \%$ and a mortality rate of $68 \%$, outcomes of the FAST method were substantially better. Compared with the standard Penumbra System, ${ }^{10}$ outcomes appeared to be slightly better, particularly with respect to mortality.

The interpretation of clinical outcomes in our cohort should be cautious. In contrast to cohorts in Western countries, the rate of atherosclerosis among stroke etiologies in our patients was relatively high, up to $50 \%$. After patients were treated with the first endovascular method, intractable severe stenosis still caused the blood flow to be stagnated, necessitating secondary angioplasty and/or stent placement, in approximately $20 \%$ of our patients. These additional procedures may delay the final revascularization 
time and worsen clinical outcomes. On this basis, investigators should consider that intracranial stenosis may be observed often when Asian patients are studied in clinical trials of endovascular treatment. Furthermore, a specific strategy of endovascular treatment should be established for Asian patients.

The FAST method is theoretically considered safe because it can avoid inadvertent hemorrhagic complications resulting from the perforation of small arteries originating from the top of the basilar artery (Fig 3). These complications may cause further decrease of mental status and new neurologic deficits. In most cases of $\mathrm{BAO}$, the distal portion above the proximal end of the occlusion is obscured. When a microwire and microcatheter must be navigated to select a posterior cerebral artery, those instruments risk perforating small arteries that are located directly beyond the basilar artery. In the FAST method, the reperfusion catheter is placed immediately proximal to the clot, and negative pressure is applied to aspirate the thrombus. Without any procedure through the thrombus, the risk of perforation is decreased (Fig 3). Recent reports on A Direct Aspiration first Pass Technique (ADAPT) have indicated that it is similar to the FAST method ${ }^{32,33}$; this technique was especially well-illustrated in a previous report. ${ }^{33}$ However, the microwire and microcatheter should be selectively traversed because this may cause a subarachnoid hemorrhage, particularly through the perforators at the top of the basilar artery.

The current study has some limitations. First, it was retrospective. A further comparative study in which both groups are randomly assigned should be performed to confirm our results. Second, both methods were not performed in the same time period. The FAST method was introduced more recently; therefore, progress in the systematic treatment for acute ischemic stroke and the development of newer intracranial microwires and microcatheters may have influenced our results. Nonetheless, major baseline characteristics and clinical events that have a substantial influence on outcomes were similar between the groups. Additionally, data were collected from 2 large hospitals and combined so that unforeseen confounding factors were less likely to have significantly affected our results. Finally, additional treatment for intracranial arterial stenosis was not considered in our analyses. Stenosis of the vertebrobasilar artery system is often observed, especially in Asians. The revascularization methods for stenosis should be applied differently. On the basis of our observations, FAST methods are better suited to embolic BAO than to atherosclerosis.

\section{CONCLUSIONS}

The FAST method by using the Penumbra reperfusion catheter demonstrated superior performance and was associated with better outcomes in patients with acute BAO compared with intraarterial fibrinolysis. Moreover, the Penumbra system has recently been enhanced. However, additional clinical trials with newer generation devices, such as Penumbra ACE and MAX, are required to confirm the conclusions of this study.

\section{REFERENCES}

1. Caplan LR, Wityk RJ, Glass TA, et al. New England Medical Center Posterior Circulation registry. Ann Neurol 2004;56:389-98

2. Hacke W, Zeumer H, Ferbert A, et al. Intra-arterial thrombolytic therapy improves outcome in patients with acute vertebrobasilar occlusive disease. Stroke 1988;19:1216-22

3. Davis SM, Donnan GA. Basilar artery thrombosis: recanalization is the key. Stroke 2006;37:2440

4. Kim HY, Chung CS, Moon SY, et al. Complete nonvisualization of basilar artery on MR angiography in patients with vertebrobasilar ischemic stroke: favorable outcome factors. Cerebrovasc Dis 2004;18:269-76

5. Pfefferkorn T, Holtmannspotter M, Schmidt C, et al. Drip, ship, and retrieve: cooperative recanalization therapy in acute basilar artery occlusion. Stroke 2010;41:722-26

6. Pfefferkorn T, Mayer TE, Opherk C, et al. Staged escalation therapy in acute basilar artery occlusion: intravenous thrombolysis and ondemand consecutive endovascular mechanical thrombectomypreliminary experience in 16 patients. Stroke 2008;39:1496-500

7. Eckert B, Kucinski T, Pfeiffer G, et al. Endovascular therapy of acute vertebrobasilar occlusion: early treatment onset as the most important factor. Cerebrovasc Dis 2002;14:42-50

8. Furlan A, Higashida R, Wechsler L, et al. Intra-arterial prourokinase for acute ischemic stroke: the PROACT II study-a randomized controlled trial. Prolyse in Acute Cerebral Thromboembolism. JAMA 1999;282:2003-11

9. Schonewille WJ, Wijman CA, Michel P, et al. Treatment and outcomes of acute basilar artery occlusion in the Basilar Artery International Cooperation Study (BASICS): a prospective registry study. Lancet Neurol 2009;8:724-30

10. Roth C, Mielke A, Siekmann R, et al. First experiences with a new device for mechanical thrombectomy in acute basilar artery occlusion. Cerebrovasc Dis 2011;32:28-34

11. Machi P, Costalat V, Lobotesis K, et al. Solitaire FR thrombectomy system: immediate results in $\mathbf{5 6}$ consecutive acute ischemic stroke patients. J Neurointerv Surg 2012;4:62-66

12. Mordasini P, Brekenfeld C, Byrne JV, et al. Technical feasibility and application of mechanical thrombectomy with the Solitaire FR revascularization device in acute basilar artery occlusion. AJNR Am J Neuroradiol 2013;34:159-63

13. Lutsep HL, Rymer MM, Nesbit GM. Vertebrobasilar revascularization rates and outcomes in the MERCI and multi-MERCI trials. $J$ Stroke Cerebrovasc Dis 2008; 17:55-57

14. Kang DH, Hwang YH, Kim YS, et al. Direct thrombus retrieval using the reperfusion catheter of the Penumbra system: forced-suction thrombectomy in acute ischemic stroke. AJNR Am J Neuroradiol 2011;32:283-87

15. Lee JS, Hong JM, Lee SJ, et al. The combined use of mechanical thrombectomy devices is feasible for treating acute carotid terminus occlusion. Acta Neurochir (Wien) 2013;155:635-41

16. Kang DH, Kim YS, Park J, et al. Rescue forced-suction thrombectomy using the reperfusion catheter of the Penumbra System for thromboembolism during coil embolization of ruptured cerebral aneurysms. Neurosurgery 2012;70(1 suppl operative):89-93, discussion 93-94

17. Kim YW, Kang DH, Hwang JH, et al. Rescue strategy for acute carotid stent thrombosis during carotid stenting with distal filter protection using forced arterial suction thrombectomy with a reperfusion catheter of the Penumbra system: a technical note. Acta Neurochir (Wien) 2013;155:1583-88

18. Arnold M, Nedeltchev K, Mattle HP, et al. Intra-arterial thrombolysis in 24 consecutive patients with internal carotid artery T occlusions. J Neurol Neurosurg Psychiatry 2003;74:739-42

19. Kucinski T, Koch C, Grzyska U, et al. The predictive value of early CT and angiography for fatal hemispheric swelling in acute stroke. AJNR Am J Neuroradiol 1998;19:839-46

20. Chandra RV, Law CP, Yan B, et al. Glasgow coma scale does not predict outcome post-intra-arterial treatment for basilar artery thrombosis. AJNR Am J Neuroradiol 2011;32:576-80

21. Eckert B, Koch C, Thomalla G, et al. Aggressive therapy with intravenous abciximab and intra-arterial rtPA and additional PTA/ stenting improves clinical outcome in acute vertebrobasilar 
occlusion: combined local fibrinolysis and intravenous abciximab in acute vertebrobasilar stroke treatment (FAST): results of a multicenter study. Stroke 2005;36:1160-65

22. Kashiwagi J, Kiyosue H, Hori Y, et al. Endovascular recanalization of acute intracranial vertebrobasilar artery occlusion using local fibrinolysis and additional balloon angioplasty. Neuroradiology 2010; $52: 361-70$

23. Barlinn K, Becker U, Puetz V, et al. Combined treatment with intravenous abciximab and intraarterial tPA yields high recanalization rate in patients with acute basilar artery occlusion. J Neuroimaging 2012;22:167-71

24. Lindsberg PJ, Mattle HP. Therapy of basilar artery occlusion: a systematic analysis comparing intra-arterial and intravenous thrombolysis. Stroke 2006;37:922-28

25. Nagel S, Schellinger PD, Hartmann M, et al. Therapy of acute basilar artery occlusion: intraarterial thrombolysis alone vs bridging therapy. Stroke 2009;40:140-46

26. Miteff F, Faulder KC, Goh AC, et al. Mechanical thrombectomy with a self-expanding retrievable intracranial stent (Solitaire $\mathrm{AB}$ ): experience in 26 patients with acute cerebral artery occlusion. AJNR Am J Neuroradiol 2011;32:1078-81

27. Mourand I, Machi P, Milhaud D, et al. Mechanical thrombectomy with the Solitaire device in acute basilar artery occlusion. J Neurointerv Surg 2014;6:200-04
28. Espinosa de Rueda M, Parrilla G, Zamarro J, et al. Treatment of acute vertebrobasilar occlusion using thrombectomy with stent retrievers: initial experience with 18 patients. AJNR Am J Neuroradiol 2013; 34:1044-48

29. Roth C, Papanagiotou P, Behnke S, et al. Stent-assisted mechanical recanalization for treatment of acute intracerebral artery occlusions. Stroke 2010;41:2559-67

30. Mourand I, Machi P, Milhaud D, et al. Mechanical thrombectomy with the Solitaire device in acute basilar artery occlusion. J Neurointerv Surg 2014;6:200-44

31. Arnold M, Nedeltchev K, Schroth G, et al. Clinical and radiological predictors of recanalisation and outcome of 40 patients with acute basilar artery occlusion treated with intra-arterial thrombolysis. J Neurol Neurosurg Psychiatry 2004;75:857-62

32. Turk AS, Frei D, Fiorella D, et al. ADAPT FAST study: a direct aspiration first pass technique for acute stroke thrombectomy. J Neurointerv Surg 2014;6:260-64

33. Turk AS, Spiotta A, Frei D, et al. Initial clinical experience with the ADAPT technique: a direct aspiration first pass technique for stroke thrombectomy. J Neurointerv Surg 2014;6:231-37

34. Adams HP Jr, Bendixen BH, Kappelle LJ, et al. Classification of subtype of acute ischemic stroke: definitions for use in a multicenter clinical trial-TOAST. Trial of Org 10172 in Acute Stroke Treatment. Stroke 1993;24:35-41 